\title{
A Review of Super Resolution and Tumor Detection Techniques in Medical Imaging
}

\author{
Fathimath Safana C. $\mathrm{K}^{\mathbf{1}}$, Sherin Mary Kuriakose ${ }^{2}$ \\ ${ }^{1}$ Student, ${ }^{2}$ Assistant Professor \\ ${ }^{1,2}$ Cochin College of Engineering and Technology, Valanchery, Kerala, India
}

\begin{abstract}
How to cite this paper: Fathimath Safana C. K | Sherin Mary Kuriakose "A Review of Super Resolution and Tumor Detection Techniques in Medical Imaging" Published in International Journal of Trend in Scientific Research and Development (ijtsrd), ISSN: 24566470, Volume-3 I Issue-3, April 2019, pp.1785-1787, URL: https://www.ijtsrd.c om/papers/ijtsrd23 525.pdf

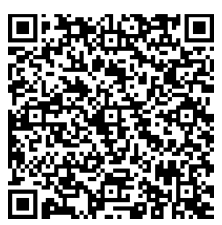
IITSRD23525
\end{abstract}

Copyright (c) 2019 by author(s) and International Journal of Trend in Scientific Research and Development Journal. This is an Open Access article distributed under the terms of the Creative Commons

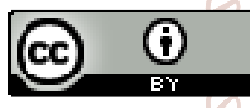
Attribution License (CC BY 4.0) (http://creativecommons.org/license s/by/4.0)

\section{INTRODUCTION}

Medical imaging also known as diagnostic imaging, through this doctors look inside your body for clues about a medical condition. A variety of machines and techniques can create pictures of the structures and activities inside your body. The type of imaging your doctor uses depends on your symptoms and the part of your body being examined. They include X rays, CT scans, Nuclear medicine scans and MRI scans ultra sound. Many imaging tests are painless and easy. Some require you to stay still for a long time inside a machine. This can be uncomfortable. Certain tests involve exposure to a small amount of radiation. Magnetic resonance imaging (MRI) is a medical imaging technique using radio waves and magnetic fields to create detailed cross-sectional images of internal organs and structures within the body. Widely used in patient analysis and medical diagnosis, MRI often reveals different information about bodily structures than can be visualized using other imaging methods such as X-ray, computed tomography (CT) or ultrasound. Medical imaging is an important diagnosis instrument to determine the presence of certain diseases. Therefore increasing the image resolution should significantly improve the diagnosis ability for corrective treatment. Furthermore, a better resolution may substantially improve automatic detection and image segmentation results. A tumor also known as neoplasm is a growth in the abnormal tissue which can be differentiated from the surrounding tissue by its structure. A tumor may lead to cancer, which is a major leading cause of death and responsible for around $13 \%$ of all deaths worldwide. Cancer incidence rate is growing at an alarming rate in the world. Great knowledge and experience on radiology are required for accurate tumor detection in medical imaging. Automation of tumor detection is required because there might be a shortage of skilled radiologists at a time of great need.

\section{OVERVIEW OF SUPER RESOLUTION}

The objective of image super-resolution is to enhance or increase the resolution of a given low-resolution image into high resolution image through some techniques like upsampling, de-blurring, de-noising, etc. In order to restore an image into a high-resolution image correctly, it is necessary to infer high frequency components of a low-resolution image. Image super-resolution is important in many applications of multimedia, such as playing a video on a higher-resolution screen. Due to some technical limitations in imaging devices and systems, like, the presence of optical distortions and lens blur, insufficient sensor sampling density and aliasing, motion blur due to low shutter speed, the presence of noise due to sensor limitations and lossy coding, super-resolution technique is actually needed. 


\subsection{Methods Related To Super Resolution.}

In this paper, we make a review on some well-known approaches or techniques related to image super resolution. From all the super resolution methods it can be categorized into three types [1]. Interpolation based, Reconstruction based and Example based. Interpolation based super resolution. Simplest way to provide super-resolution is to apply interpolation on the sampled visual data acquired from the sensor. It is one of the early super-resolution algorithms based on resampling (a mathematical technique used to create a new version of the image with a different width and/or height in pixels). This approach, for example, which is present in digital cameras via the digital zoom, ultimately relies on the operations based on linear filtering. [2] This technique has advantage of less computational complexity due to its simplicity and also real-time applications are possible. The reconstruction-based superresolution approaches [3] apply various smoothness priors and impose the constraint that when properly down sampled, the high-resolution image should reproduce the original low-resolution image. In example based methods [4] there having a database of high-resolution images corresponding to the low-resolution examples that best resembles or matches the two parts of given image and reconstruct the corresponding high-resolution version of the given image with the technique of selecting, editing and piecing together.

\subsection{Estimation Parameters of Super Resolution.}

The performance evaluation metrics of super resolution are peak signal to noise ratio (PSNR), the structural similarity and mean square error [5]. PSNR is the ratio between the maximum possible power of a signal and the power of corrupting noise that affects the fidelity of its representation. Because many signals have a very wide dynamic range, PSNR is usually expressed in terms of the logarithmic decibel scale. Structural similarity is the index is a method for predicting the perceived quality of digital television and cinematic pictures, as well as other kinds of digital images and videos. Mean square error is the measures the average of the squares of the errors that is, the average squared difference between the estimated values and what is estimated.

\section{OVERVIEW OF TUMOR DETECTION}

Nowadays, brain tumor has become one of the main causes for increasing mortality among children and adults. This facts increase the importance of the researches on the tumor detection and this will present the opportunity for doctors to help save lives by detecting the disease earlier and perform necessary actions. Varieties of image processing techniques are available to be applied on various imaging modalities for tumor detection that will detect certain features of the tumors such as the shape, border, calcification and texture. These features will make the detection processes more accurate and easier as there are some standard characteristics of each feature for a specific tumor

\subsection{Methods Related To Tumor Detection}

Deep learning [6] is a machine learning technique that teaches computers to do what comes naturally to humans: learn by example. The second common cancer is Breast cancer in the women population. Detection of breast cancer [7] in early stage increases chances for treatment. Mammography is the best screening instrument that uses low dose X-rays to find tumors in a breast image. Mammography images suffer from low quality and contrast. images are preprocessed by morphological filters. ROTs in images are determined by segmentation. Then, Extracted features fed to K-NN. Preprocessing reduces the glandular tissues and noises. In this stage, use two types of morphological filters, the opening filter and closing filter that are used in the mammography gray images. Then Extraction of ROls to segment regions of tumor in each image. In this paper [8] data mining methods are used for classification of MRI images. A new hybrid technique based on the support vector machine (SVM) and fuzzy c-means for brain tumor classification is proposed [9]. The purposed algorithm is a combination of support vector machine (SVM) and fuzzy cmeans, a hybrid technique for prediction of brain tumor. The enhanced images are fed to a pre-trained convolutional neural network (CNN) which is a member of deep learning models. The CNN classifier, [10] which is trained by large number of training samples, distinguishes between melanoma and benign cases.

\subsection{Estimation Parameters of Tumor Detection}

Accuracy, sensitivity and specificity are main parameters to estimate the tumor detection. These parameters are calculated through some terms like TP, be the True Positive which is number of pixels correctly identified as tumor. FP is False Positive; it is number of pixels incorrectly identified as tumor. Similarly TN the True Negative is number of pixels correctly identified as healthy and FN is False Negative is a number of pixels incorrectly identified as healthy.

\section{CONCLUSION}

This paper described the various super resolution methods and different tumor detection methods. The main estimation parameters of super resolution are peak signal to noise ratio, structural similarity and mean square error. Accuracy, sensitivity and specificity are the main parameters of tumor detection techniques. Medical imaging is an important diagnosis instrument to determine the presence of certain diseases. Therefore increasing the image resolution should significantly improve the diagnosis ability for corrective treatment. Furthermore, a better resolution may substantially improve automatic detection and image segmentation results.

\section{REFERENCES}

[1]. Nirali Harsoda, Namarata Joshi. "A Review on Image Super Resolution Techniques". International Journal of Engineering Research and Technology (IJERT), Vol. 3, Issue 2, February 2014.

[2]. Jonathan Sachs. "Image Resampling". Digital Light andColor, 2011.

[3]. W. S. Tam, C. W. Kok, W. C. Siu. "A modified edge directed interpolation for images".Journal of Electronic Imaging, 19(1), 013011:1-20, 2010.

[4]. W. T. Freeman, T. R. Jones, E. C. Pasztor. "Examplebased super-resolution". IEEE Computer Graphics and Applications, pp. 56 - 65, Vol. 22, Issue 2, 2002.

[5]. X. J. Kim, J. Kwon Lee, and K. Mu Lee, "Accurate image super-resolution using very deep convolutional networks," in Proceedings of the IEEE Conference on Computer Vision and Pattern Recognition, pp. 16461654. 2016.

[6]. Y. LeCun, Y. Bengio, and G. Hinton, "Deep learning," Nature, vol. 521, no. 7553, pp. 436-444, 2015 
[7]. Fatemeh shirazi, Esmat rashedi, "Feature Weighting For Cancer Tumor Detection In Mammography Images Using Gravitational Search Algorithm", 6th International Conference on Computer and Knowledge Engineering (ICCKE 2016), October 20-21, 2016

[8]. Parveen, Amritpal singh, "Detection of Brain Tumor in MRI Images, Using Combination of Fuzzy C-Means and SVM," 2nd International Conference on Signal Processing and Integrated Network s2015
[9]. E. Nasr-Esfahani, S. Samavi, N. Karimi, S.M.R. Soroushmehr, M.H. Jafari, K. Ward, K. Najarian "Melanoma Detection by Analysis of Clinical Images Using Convolutional Neural Network, IEEE, pp. 770778.

[10]. Sergio Pereira, Adriano Pinto, Victor Alves and Carlos A. Silva, "Brain Tumor Segmentation using Convolutional Neural Networks in MRI Images," IEEE Transactions on Medical Imaging, vol. 49, no. 11, pp. $1875-1883,2016$

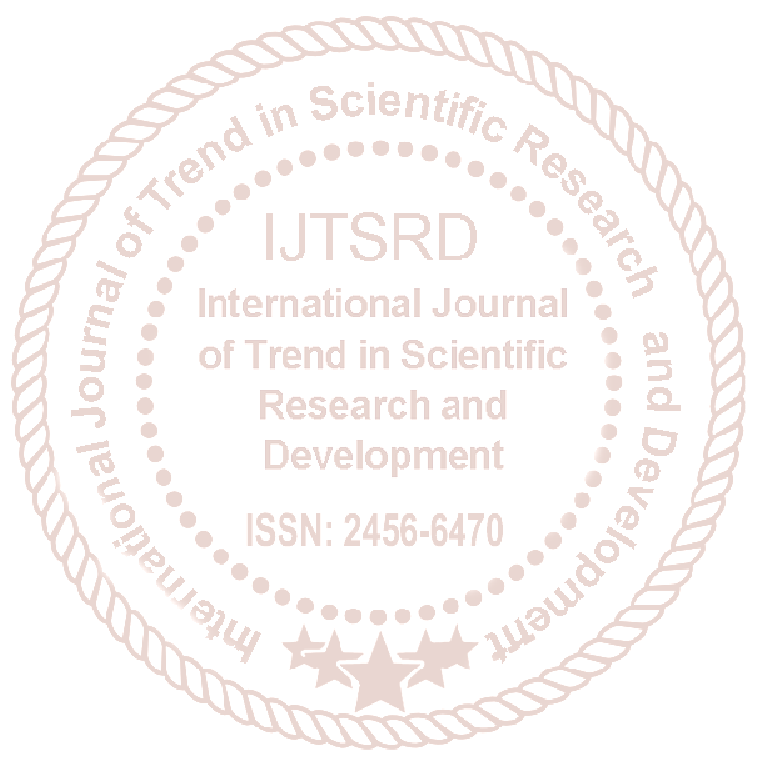

